\title{
Relationship between Awareness of Healthy Lifestyle and Perception of Breast Cancer among Women of Reproductive
} Age

\author{
Yeliz VARIŞOĞLU, PhD and Pınar IRMAK VURAL (D, PhD \\ Department of Nursing, Health Science Faculty, Istanbul Medipol University, Turkey
}

*Corresponding author: PInar IRMAK VURAL, Department of Nursing, Health Science Faculty, Istanbul Medipol University, Ekinciler Street Nu.19 Kavacık-Beykoz 34810 Istanbul, Turkey, Tel: +90-505-663-72-16, Fax: +90-212-531-75-55

\begin{abstract}
Objectives: The aim of this study was to determine the relationship between awareness of healthy lifestyle and breast cancer perception among women of reproductive age.

Methods: This descriptive co relational study was conducted on 642 women. Data were collected using the sociodemographic data form, the Healthy Lifestyle Awareness Scale (HLAS), and the Breast Cancer Perception Scale (BCPS). A moderate, significant, and positive correlation was found between the HLAS total score and the scores obtained from the BCPS sub-dimensions of perceived knowledge and perceived treatment belief.

Results: The regression analysis on the relationship between the scores of HLAS's change sub-dimension and BCPS sub-dimensions showed that as the scores of perceived knowledge and perceived treatment belief sub-dimensions increased, the change scores increased significantly. The explanatory value of these findings was $27.1 \%$.

Conclusion: It has been demonstrated that women with advantages of high educational and economic levels, an income-generating job, and working in urban areas have higher perceived knowledge and treatment belief levels and low levels of fear with regard to breast cancer.
\end{abstract}

\section{Keywords}

Breast cancer, Healthy lifestyle awareness, Women health, Cancer perception

\section{Introduction}

The improvement and maintenance of health are associated with the adoption of healthy lifestyle behavior. A healthy lifestyle is a range of behaviors complying with the health status of the individual to control all activities impacting on their wellbeing and organize their daily activities. Walker, et al. defined healthy lifestyle behaviors as those that help maintain and improve the well-being of the individual [1].

Mindfulness refers to focusing the attention on the present moment and accepting whatever is happening and whatever is felt in that moment, in a non-judgmental way. Mindfulness of the individual indicates that wellbeing and awareness are connected. The importance of the experienced state of awareness for the purpose of achieving and improving general well-being has been addressed by several philosophical, spiritual, and psychological schools. Thus, the role of the awareness of the need to change the lifestyle is noted in helping the individual to maintain their wellbeing and in protecting them from developing diseases. Because mindfulness aims to avoid automatic thoughts and habits, it plays an important role in life leading the individual to give up unhealthy behavioral patterns and to develop conscious behavior $[2,3]$.

A healthy lifestyle includes a set of acquired skills. Many diseases encountered in adulthood can be prevented by leading an effective lifestyle [4]. Examples of such diseases include heart diseases, diabetes, chronic respiratory problems, and cancer, all of which are the most common causes of death [5]. Cancer cases of genetic origin account for only $5-10 \%$ of all cancer cases. It is reported that cancer is mostly caused by

Citation: VARIŞOĞLU Y, VURAL PI (2022) Relationship between Awareness of Healthy Lifestyle and Perception of Breast Cancer among Women of Reproductive Age. Int J Oncol Res 5:035. doi. org/10.23937/2643-4563/1710035

Accepted: January 29, 2022: Published: January 31, 2022

Copyright: (C) 2022 VARIŞOĞLU Y, et al. This is an open-access article distributed under the terms of the Creative Commons Attribution License, which permits unrestricted use, distribution, and reproduction in any medium, provided the original author and source are credited. 
environmental and lifestyle factors [6]. It is highlighted that most diseases of adult ages including cancer, heart attack, stroke, diabetes, and chronic respiratory problems can be prevented by adopting healthy lifestyle in the early years of life $[7,8]$.

Breast cancer is the most common type of cancer and the most common cause of death among women across the world and in Turkey. The incidence of breast cancer in Turkey is over $50 / 100,000$. The incidence of breast cancer has increased approximately 2.5 times over the last 25 years $[9,10]$. The reasons for this increase may include lifestyle changes (obesity, inactivity, infertility, late age at childbirth ( $>35$ years), short term breastfeeding, early menarche, late menopause, longterm use of birth control pills and receiving treatment for menopause, etc.), aging of the population, awareness (warnings from the media, patient information and referrals for screening mammography in breast diseases and menopause outpatient clinics, women's increasing levels of awareness and education status, etc.), increasing numbers of mammography performed at irregular intervals, and population growth [11]. According to the literature, leading a healthy lifestyle (avoiding obesity, engaging in regular workouts, adopting a balanced diet, and avoiding alcohol and longterm hormone replacement therapy) and using drugs to prevent breast cancer reduce the risk of breast cancer $50 \%$ [12].

The significant increase in survival rates after breast cancer treatment and the success achieved in the fight against the disease are based on two major factors: Improved efficacy of local and systemic treatment methods and early diagnosis. In addition to advanced imaging methods, breast self-examination and clinical breast examination play important roles in the early diagnosis. The National Cancer Institute of USA recommends that women at average risk of breast cancer should undergo breast examinations starting in their twenties as part of their routine health checkup schedules. After the age of 40 , it is recommended to include mammography in annual breast examination programs [11].

It is suggested that women's healthy lifestyles are correlated to their perceptions of breast cancer and to its early detection. Therefore, it is important to determine the patterns of women's perceptions of breast cancer [13]. Perception is the process of the individual's evaluation of recent and past experiences and the achievement of a sense of the new whole [14]. Determining how breast cancer perceptions impact on healthy lifestyle awareness and early detection behaviors is also important to develop such behaviors [13]. In this context, the aim of this study was to determine the relationship between awareness of healthy lifestyle and breast cancer perception among women of reproductive age.

\section{Methods}

\section{Purpose and type of study}

The purpose of this descriptive co relational study was to determine the relationship between awareness of healthy lifestyle and breast cancer perception among women of reproductive age.

\section{Population and sample of study}

The population of this study consisted of women who were Turkish citizens, literate, at ages of 18-49 years, and were not diagnosed with breast cancer previously. The sample of the study consisted of 642 women who were Turkish citizens, literate, at ages of 18-49 years, were not diagnosed with breast cancer previously, and agreed to participate in the study.

\section{Data collection tools}

Socio-demographic data form, Healthy Lifestyle Awareness Scale, and Breast Cancer Perception Scale were used to collect the study data. This study was conducted between May 2021 and July 2021 by delivering the link of the data collection tools, which were developed on the Google Forms application, to individuals volunteering to participate in the study.

The socio-demographic data form was developed by the researchers. The form included 10 questions about socio-demographic information.

The Healthy Lifestyle Awareness Scale (HLAS) measures individuals' awareness of healthy living, consisting of 4 sub-dimensions (socialization, responsibility, change, nutrition) and 15 items. The lowest score of the scale is 15 and the highest one is 75. A high score on the scale indicates a high level of awareness of healthy living. Cronbach's alpha value of HLAS was 0.813 [3]. In this study, the Cronbach's alpha value was found to be 0.88 .

The Breast Cancer Perception Scale (BCPS) offers a multidimensional evaluation of breast cancer perception in women. The dimensions include healthy lifestyle behaviors, breast cancer diagnostic behaviors, family history, traumatic experiences related to breast cancer, and breast cancer knowledge levels. BCPS can be used to evaluate and understand the relationship between breast cancer and breast cancer diagnostic behaviors, such as breast self-examination, clinical breast examination, getting mammography, and maintaining healthful behaviors like diet, exercise, and healthy eating. BCPSitems are scored on a 5-point Likert scale, consisting of 24 items and 6 sub-dimensions (perceived knowledge, perceived treatment belief, the perceived need for a health check, perceived stigma, perceived fear, and perceived risk). A total score was not defined. Items 9, 10, 11, 12, and 13 are reverse coded. Increasing scores indicate increased levels of perception about the respective sub-dimension. Cronbach's alpha reliability coefficients of the scale sub- 
dimensions were in the range of $0.815-0.950$ [13]. In this study, Cronbach's alpha reliability coefficient of the scale sub-dimensions was 0.85 .

Table 1: Participants' descriptive characteristics $(N=642)$.

\begin{tabular}{|l|l|}
\hline Descriptive characteristics & Mean \pm SD \\
\hline Age & $34.32 \pm 11.42$ \\
\hline Number of pregnancy & $2.21 \pm 1.77$ \\
\hline Number of birth & $1.91 \pm 1.45$ \\
\hline BMI & $25.24 \pm 4.96$ \\
\hline Duration of breastfeeding (month) & $13.59 \pm 12.03$ \\
\hline Educational status & N (\%) \\
\hline Primary school & $140(21.8)$ \\
\hline Secondary school & $77(12.0)$ \\
\hline High school & $150(23.4)$ \\
\hline University and higher & $275(42.8)$ \\
\hline Marital status & \\
\hline The married & $391(60.1)$ \\
\hline Single & $251(39.1)$ \\
\hline Working status & \\
\hline Employed & $301(46.9)$ \\
\hline Unemployed & $331(51.6)$ \\
\hline Retired & $10(1.6)$ \\
\hline Economic status & \\
\hline Less than income & $73(11.5)$ \\
\hline Equal to income and expenditure & $412(64.2)$ \\
\hline Income higher than expenditure & $157(24.3)$ \\
\hline Place of longest residence & \\
\hline City & $469(73.1)$ \\
\hline Town & $122(19.0)$ \\
\hline Village & $51(7.9)$ \\
\hline Smoking & \\
\hline Yes & $136(21.2)$ \\
\hline No & $506(78.8)$ \\
\hline & \\
\hline
\end{tabular}

Note: SD: Standart Deviation

\section{Data analysis}

SPSS 22 package software was used for statistical analysis. A p-value of $<0.05$ was accepted as statistically significant for all results. Descriptive statistics including frequency, percentage, and mean were used to summarize data. The t-test and ANOVA were used to compare independent groups. The Durbin-Watson test, a linear regression test, was used for further analysis.

\section{Ethical considerations of the study}

Informed consent was obtained from the individuals, who volunteered to participate in this study. Ethics approval was obtained from the Republic of Turkey, Ministry of Health, General Directorate of Health Services and from the Non-Interventional Ethics Committee of the respective university (Number: E-10840098-772.02-2673/588/03.06.2021). An online consent statement was obtained from each volunteer participating in the study.

\section{Results}

The mean age of the women participating in the study was $34.32 \pm 11.42$ years, the mean BMI was 25.24 \pm 4.96 , and the mean number of pregnancies was 2.21 \pm 1.77 . Of the participants, $42.8 \%$ attended university or higher educational programs, $46.9 \%$ worked in a job that generated income, $64.2 \%$ had an average income level, and $73.1 \%$ lived in urban areas for the most of their lives. In the study, $60.1 \%$ of the women were married and $78.8 \%$ were non-smokers (Table 1 ).

Table 2 shows the mean total scores and the scores of all sub-dimensions of the participants in BCPS and HLAS. In the BCPS, the mean score of perceived knowledge was $11.57 \pm 3.48$, the mean score of perceived stigma was $8.59 \pm 2.94$, and the mean score of perceived risk was $9.13 \pm 2.19$. The mean total HLAS score was 58.47 \pm 8.01 (Table 2).

A moderate, significant, and positive correlation

Table 2: Participants' scales and subscales mean scores $(\mathrm{N}=642)$.

\begin{tabular}{|l|l|l|}
\hline The Breast Cancer Perception Scale & Mean \pm SD & Min-Max \\
\hline Perceived knowledge & $11.57 \pm 3.48$ & $4-20$ \\
\hline Perceived treatment belief & $21.91 \pm 2.98$ & $5-25$ \\
\hline Perceived need for healthcheck & $11.08 \pm 3.56$ & $4-20$ \\
\hline Perceived stigma & $8.59 \pm 2.94$ & $4-18$ \\
\hline Perceived fear & $15.10 \pm 3.79$ & $4-20$ \\
\hline Perceived risk & $9.13 \pm 2.19$ & $3-15$ \\
\hline The Healthy Life Awareness Scale & & \\
\hline Change & $20.93 \pm 2.91$ & $6-25$ \\
\hline Socialization & $14.94 \pm 3.03$ & $4-20$ \\
\hline Responsibility & $11.87 \pm 2.03$ & $3-15$ \\
\hline Nutrition & $10.71 \pm 2.58$ & $3-15$ \\
\hline Total Healthy Life Awareness & $58.47 \pm 8.01$ & $21-75$ \\
\hline
\end{tabular}

Notes: SD: Standart Deviation; Min: Minimum; Max: Maximum 
Table 3: Relationship between scales and subscales scores.

\begin{tabular}{|c|c|c|c|c|c|}
\hline \multirow{2}{*}{$\begin{array}{l}\text { The Breast Cancer Perception } \\
\text { Scale }\end{array}$} & \multicolumn{5}{|c|}{ The Healthy Life Awareness Scale } \\
\hline & Change & Socialization & Responsibility & Nutrition & Total \\
\hline \multirow{2}{*}{ Perceived knowledge } & r: $0.191^{\star *}$ & r: $0.279^{* *}$ & $r: 0.293^{* *}$ & r: $0.324^{* *}$ & $r: 0.354^{* *}$ \\
\hline & p: 0.000 & p: 0.000 & p: 0.000 & p: 0.000 & p: 0.000 \\
\hline \multirow{2}{*}{ Perceived treatment belief } & r: $0.491^{\star *}$ & $r: 0.245^{\star \star}$ & r: $0.449^{* *}$ & $\mathrm{r}: 0.221^{\star *}$ & $\mathrm{r}: 0.457^{\star *}$ \\
\hline & p: 0.000 & $\mathrm{p}: 0.000$ & $\mathrm{p}: 0.000$ & p: 0.000 & p: 0.000 \\
\hline \multirow{2}{*}{ Perceived need for healthcheck } & r: 0.062 & r: $0.199^{* *}$ & $r: 0.138^{\star *}$ & $r: 0.280^{* *}$ & $\mathrm{r}: 0.224^{\star *}$ \\
\hline & $\mathrm{p}: 0.117$ & $\mathrm{p}: 0.000$ & p: 0.000 & p: 0.000 & p: 0.000 \\
\hline \multirow{2}{*}{ Perceived stigma } & $\mathrm{r}:-0.230^{* *}$ & $\mathrm{r}:-0.061$ & $\mathrm{r}:-0.232^{* *}$ & $\mathrm{r}:-0.181^{* *}$ & $\mathrm{r}:-0.224^{* *}$ \\
\hline & p: 0.000 & p: 0.124 & p: 0.000 & p: 0.000 & p: 0.000 \\
\hline \multirow{2}{*}{ Perceived fear } & r: $0.087^{*}$ & r: $0.110^{* *}$ & r: 0.022 & r: $-0.107^{\star *}$ & r: 0.044 \\
\hline & p: 0.028 & p: 0.005 & $\mathrm{p}: 0.582$ & p: 0.007 & $\mathrm{p}: 0.264$ \\
\hline \multirow{2}{*}{ Perceived risk } & r: -0.029 & r: $0.077^{*}$ & r: 0.017 & r: -0.017 & r: 0.017 \\
\hline & p: 0.455 & p: 0.049 & p: 0.669 & p: 0.666 & p: 0.66 \\
\hline
\end{tabular}

Notes: ${ }^{* *}$ Correlations is significant at the 0.01 level (2-tailed); ${ }^{*}$ Correlation is significant at the 0.05 level (2-tailed)

was found between the HLAS total score and the scores obtained from the BCPS sub-dimensions of perceived knowledge and perceived treatment belief (Table 3).

The comparison of the socio-demographics and the mean BCPS and HLAS scores of the study participants is presented in Table 4. It was found out that as the education level of the participant increased, the scores of perceived knowledge, perceived treatment belief, and the perceived need for a health check increased and the scores of perceived stigma and perceived fear decreased among the BCPS sub-dimensions. It was determined that education levels significantly affected HLAS total scores and the scores of change, responsibility, and nutrition sub-dimensions $(p<0.05)$. It was found out that as the education level increased, the scores of perceived knowledge, treatment belief, and need for a health check, and the scores of perceived stigma and perceived fear decreased. In parallel to this finding, it was found out that HLAS scores increased as the level of the education status increased (Table 4).

The examination of the correlation between the scores of BCPS and HLAS and the marital status showed that the perceived stigma, fear and risk scores were significantly higher in unmarried participants compared to married ones $(p<0.05)$. It was determined that marital status did not affect HLAS total scores $(p>0.05)$ (Table 4).

HLAS total scores and the responsibility and change sub-dimension scores of women working in an incomegenerating job were significantly higher compared to those who were retired or unemployed. Women working in an income-generating job had also higher perceived treatment belief scores and lower perceived stigma scores in BCPS compared to other participants (Table 4).

The comparison of the scale scores by the economic level showed that as the economic level increased, the total score and the sub-dimension scores of change, responsibility, and nutrition in HLAS increased significantly $(p<0.001)$. It was found out that as the economic level increased, the sub-dimension scores of perceived knowledge, perceived treatment belief, and the perceived need for a health check increased and the perceived stigma sub-dimension scores decreased in BCPS (Table 4).

The comparison of the scale scores of the participants by the area where they lived for the longest time showed that the women living in urban areas had higher scores of perceived knowledge, perceived treatment, and the perceived need for a health check scores and lower scores of perceived stigma in BCPS plus higher HLAS total scores compared to those living in rural areas. It was determined that the smoking status of the participants did not affect the BCPS and HLAS scores (Table 4).

In the regression analysis examining the relationship between the HLAS change sub-dimension scores and the BCPS sub-dimension scores of the participants (Table 5), it was found that the change sub-dimension scores increased significantly with increasing perceived knowledge $(B=0.162, p<0.001)$ and perceived treatment belief ( $B=0.451, p<0.001$ ) sub-dimension scores and with decreasing the perceived need for a health check, perceived stigma, and perceived risk subdimension scores. The explanatory value was $27.1 \%$ in model no 1.

In the regression analysis examining the relationship between the socialization scores in HLAS and the subdimension scores in BCPS (Table 5), model no 2 showed a significant difference ( $F: 17.704, p<0.001, R^{2}: 0.143$ ).

The regression analysis examining the relationship between HLAS responsibility scores and BCPS subdimension scores (Table 5) showed that as the perceived 


\begin{tabular}{|c|c|c|c|c|c|c|c|c|c|c|c|c|c|c|c|c|c|c|c|c|c|}
\hline$\frac{0}{\pi}$ & |ełol & & $\begin{array}{l}\infty \\
\infty \\
\infty \\
+1 \\
0 \\
0 \\
0 \\
0 \\
0\end{array}$ & 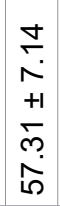 & $\begin{array}{l}\tilde{N} \\
\infty \\
\infty \\
+1 \\
\\
\\
\tilde{D}\end{array}$ & 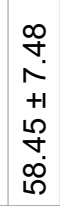 & 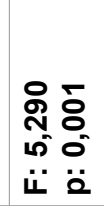 & & 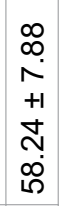 & 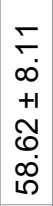 & 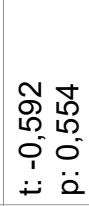 & & $\begin{array}{l}8 \\
0 \\
+1 \\
+1 \\
\hat{N} \\
\infty \\
0 \\
0\end{array}$ & $\begin{array}{l}\stackrel{0}{0} \\
\infty \\
+1 \\
\hat{n} \\
\infty \\
\infty \\
\infty \\
\infty\end{array}$ & 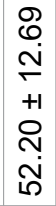 & 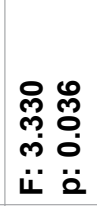 & & 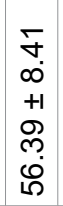 & 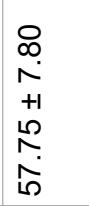 & $\begin{array}{l}0 \\
\vdots \\
+1 \\
+1 \\
m \\
m \\
\vdots \\
0\end{array}$ & 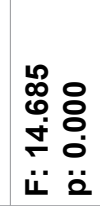 \\
\hline 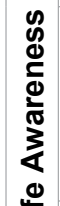 & uo!̣!!ฺฺnN & & $\begin{array}{l}5 \\
i \\
i \\
+1 \\
\infty \\
\infty \\
0\end{array}$ & 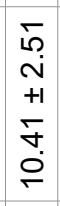 & $\begin{array}{l}\overline{5} \\
i \\
+1 \\
+1 \\
0 \\
0 \\
0 \\
\end{array}$ & 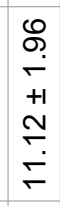 & 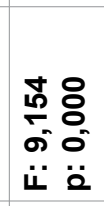 & & $\begin{array}{l}8 \\
0 \\
N \\
+1 \\
0 \\
0 \\
0 \\
0 \\
0\end{array}$ & $\begin{array}{l}0 \\
0 \\
i \\
+1 \\
+1 \\
0 \\
0 \\
0 \\
\end{array}$ & \begin{tabular}{ll}
0 & \multirow{F}{*}{} \\
6 & 5 \\
0 & 0 \\
0 & 0 \\
-1 & 0
\end{tabular} & & 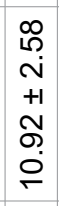 & $\begin{array}{l}\hat{L} \\
\stackrel{1}{ } \\
+1 \\
0 \\
b \\
0 \\
0\end{array}$ & 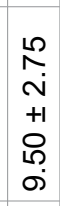 & 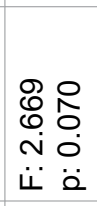 & & $\begin{array}{l}d \\
i \\
i \\
+1 \\
o \\
o \\
0\end{array}$ & 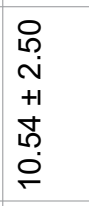 & 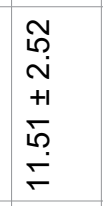 & 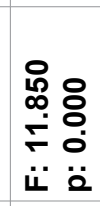 \\
\hline 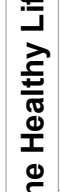 & Кł!!!q!suodsəy & & 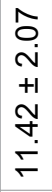 & 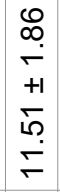 & 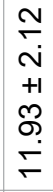 & 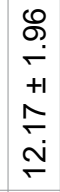 & 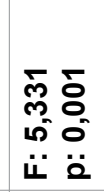 & & $\begin{array}{l}\hat{\sigma} \\
\dot{+} \\
+1 \\
\bar{\sigma} \\
\stackrel{5}{\leftarrow}\end{array}$ & $\begin{array}{l}\infty \\
0 \\
i \\
+1 \\
0 \\
\infty \\
\check{\Gamma} \\
亡\end{array}$ & 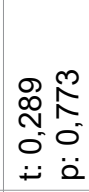 & & 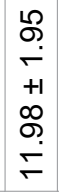 & 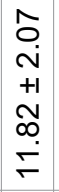 & $\begin{array}{l}+ \\
1 \\
i \\
+1 \\
+ \\
+ \\
0 \\
0\end{array}$ & 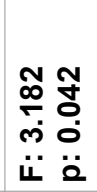 & & 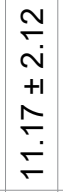 & 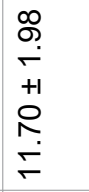 & $\begin{array}{l}8 \\
\stackrel{8}{+} \\
+1 \\
\dot{+} \\
\stackrel{+}{+}\end{array}$ & 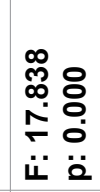 \\
\hline 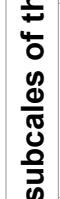 & uo!̣ez!|!!̣os & & $\begin{array}{l}\stackrel{N}{\sim} \\
\dot{m} \\
++ \\
\stackrel{N}{N} \\
\dot{J}\end{array}$ & 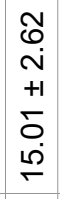 & 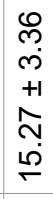 & $\begin{array}{l}\bar{\delta} \\
i \\
+1 \\
0 \\
0 \\
\dot{T}\end{array}$ & 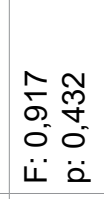 & & $\begin{array}{l}\check{\Gamma} \\
\dot{m} \\
+1 \\
\tilde{O} \\
\dot{j} \\
\dot{J}\end{array}$ & 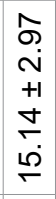 & $\begin{array}{ll} & \infty \\
0 & 0 \\
0 & 0 \\
i & 0 \\
i & 0\end{array}$ & & $\begin{array}{l}8 \\
\dot{j} \\
+1 \\
\dot{T} \\
\dot{J} \\
\dot{J}\end{array}$ & 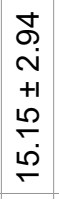 & 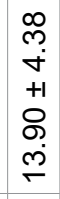 & 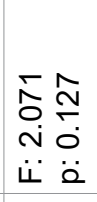 & & $\begin{array}{l}10 \\
\dot{m} \\
+1 \\
\infty \\
0 \\
10 \\
\dot{T}\end{array}$ & 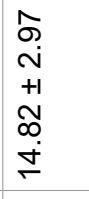 & 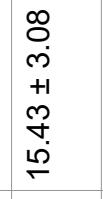 & 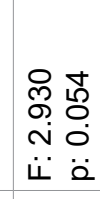 \\
\hline 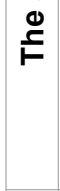 & әбиечว & & 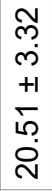 & 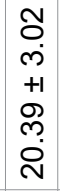 & $\begin{array}{l}\bar{\sigma} \\
i \\
+1 \\
+ \\
\sigma \\
\dot{N}\end{array}$ & $\begin{array}{l}\overline{6} \\
i \\
+1 \\
\stackrel{N}{N} \\
\grave{N}\end{array}$ & 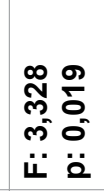 & & $\begin{array}{l}\bar{\lambda} \\
i \\
+1 \\
\bar{\delta} \\
\dot{i}\end{array}$ & 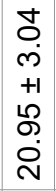 & \begin{tabular}{ll}
0 & \multirow{2}{N}{} \\
& 0 \\
0 & 0 \\
1 & 0 \\
$i$ & 0
\end{tabular} & & 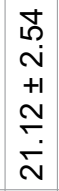 & $\begin{array}{l}\stackrel{N}{c} \\
\dot{m} \\
+1 \\
\infty \\
\infty \\
\dot{N}\end{array}$ & $\begin{array}{l}\infty \\
\infty \\
+ \\
+1 \\
+1 \\
0 \\
+ \\
\infty \\
\infty\end{array}$ & 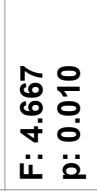 & & $\begin{array}{l}\infty \\
i \\
i \\
+1 \\
0 \\
0 \\
\dot{i} \\
\dot{N}\end{array}$ & 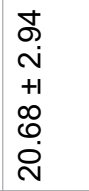 & $\begin{array}{l}\hat{N} \\
i \\
+1 \\
N \\
N \\
\bar{N}\end{array}$ & 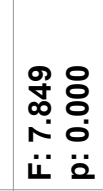 \\
\hline & YS!ı рәભ!əכגәd & & $\begin{array}{l}\tilde{m} \\
\sim \\
+1 \\
+1 \\
\tilde{m} \\
\tilde{\sigma}\end{array}$ & 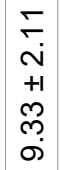 & $\begin{array}{l}\infty \\
\check{1} \\
+1 \\
\sigma \\
\sigma \\
\sigma\end{array}$ & $\begin{array}{l}\hat{\gamma} \\
\text { ì } \\
+1 \\
\Delta \\
\dot{\sigma} \\
\infty\end{array}$ & 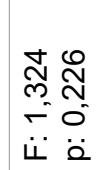 & & $\begin{array}{l}\text { N } \\
\text { N } \\
+1 \\
N \\
\mathbb{N} \\
\infty\end{array}$ & 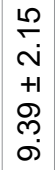 & 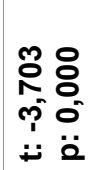 & & 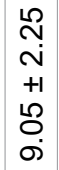 & 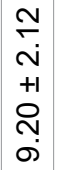 & 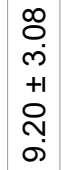 & 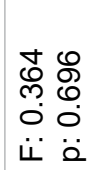 & & 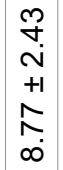 & $\begin{array}{l}8 \\
0 \\
N \\
+1 \\
+ \\
\\
\dot{\sigma}\end{array}$ & 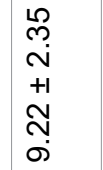 & 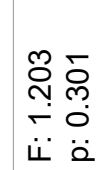 \\
\hline 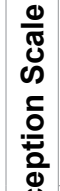 & 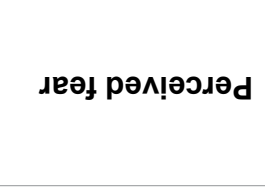 & & 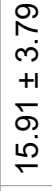 & 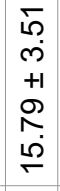 & $\begin{array}{l}0 \\
r \\
m \\
+1 \\
\sigma \\
\sigma \\
\dot{\sigma}\end{array}$ & $\begin{array}{l}\bar{\infty} \\
\infty \\
+1 \\
+\infty \\
\dot{\sigma}\end{array}$ & 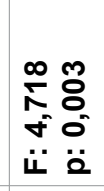 & & $\begin{array}{l}\stackrel{N}{N} \\
\dot{m} \\
+1 \\
\tilde{\sigma} \\
\dot{j}\end{array}$ & 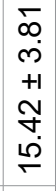 & 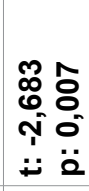 & & $\begin{array}{l}\bar{\sigma} \\
\dot{m} \\
+1 \\
\tilde{\sigma} \\
\dot{\leftarrow} \\
\dot{\leftarrow}\end{array}$ & $\begin{array}{l}\infty \\
0 \\
\infty \\
+1 \\
+\infty \\
\sim \\
\sim \\
\stackrel{\infty}{\sim}\end{array}$ & $\begin{array}{l}\mathscr{8} \\
\infty \\
\dot{1} \\
+1 \\
0 \\
0 \\
\dot{+} \\
+\end{array}$ & 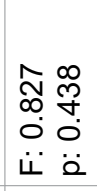 & & 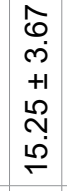 & 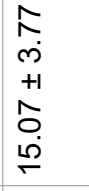 & $\begin{array}{l}8 \\
\dot{m} \\
+1 \\
+1 \\
\stackrel{0}{0} \\
\dot{b}\end{array}$ & $\begin{array}{ll}0 & m \\
0 & \tilde{g} \\
0 & 0 \\
\dot{0} & 0 \\
\dot{u} & 0\end{array}$ \\
\hline 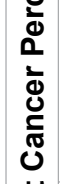 & $\begin{array}{r}\text { eu6!!!s } \\
\text { рәм!әэдәd }\end{array}$ & & $\begin{array}{l}10 \\
\infty \\
N \\
+1 \\
\infty \\
\infty \\
0 \\
\infty\end{array}$ & $\begin{array}{l}0 \\
\infty \\
N \\
+1 \\
+1 \\
N \\
\end{array}$ & $\begin{array}{l}\mathfrak{N} \\
\sim \\
+1 \\
\infty \\
\infty \\
\infty \\
\infty\end{array}$ & $\begin{array}{l}\vec{D} \\
\dot{N} \\
+1 \\
0 \\
\infty \\
\infty \\
\end{array}$ & 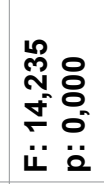 & & $\begin{array}{l}J \\
\dot{m} \\
+1 \\
\tilde{\sigma} \\
\end{array}$ & $\begin{array}{l}\bar{\infty} \\
i \\
+1 \\
\dot{J} \\
\dot{\sigma}\end{array}$ & 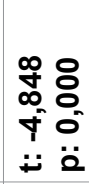 & & $\begin{array}{l}L \\
\varrho \\
N \\
+1 \\
10 \\
\stackrel{L}{\infty} \\
\infty\end{array}$ & $\begin{array}{l}\hat{\infty} \\
\infty \\
N \\
+1 \\
\hat{\sigma} \\
\infty \\
\infty\end{array}$ & $\begin{array}{l}\tilde{O} \\
\dot{n} \\
+1 \\
\stackrel{2}{0} \\
0\end{array}$ & 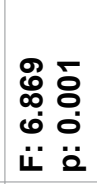 & & $\begin{array}{l}\hat{N} \\
\tilde{m} \\
+1 \\
m \\
\tilde{\sigma}\end{array}$ & $\begin{array}{l}\sigma \\
\sim \\
N \\
+1 \\
0 \\
\infty \\
\infty\end{array}$ & 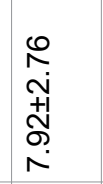 & 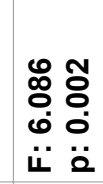 \\
\hline 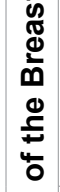 & 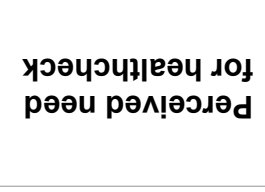 & & $\begin{array}{l}10 \\
10 \\
0 \\
+1 \\
20 \\
0 \\
0 \\
0\end{array}$ & $\begin{array}{l}0 \\
+ \\
0 \\
0 \\
+1 \\
1 \\
0 \\
0 \\
0 \\
0\end{array}$ & 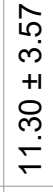 & 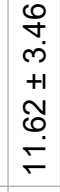 & 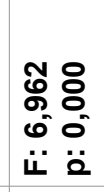 & & 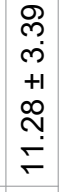 & $\begin{array}{l}0 \\
0 \\
\dot{\infty} \\
+1 \\
1 \\
0 \\
0 \\
0\end{array}$ & 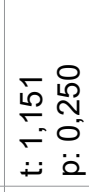 & & $\begin{array}{l}\tilde{\omega} \\
m \\
+1 \\
m \\
m \\
\stackrel{+}{=}\end{array}$ & $\begin{array}{l}9 \\
+ \\
0 \\
+1 \\
\infty \\
\infty \\
0 \\
0\end{array}$ & 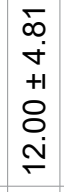 & 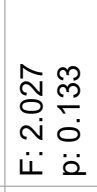 & & $\begin{array}{l}\dot{N} \\
\tilde{n} \\
+1 \\
\tilde{D} \\
\dot{\sigma}\end{array}$ & 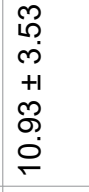 & 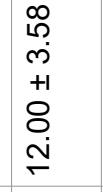 & 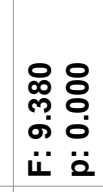 \\
\hline 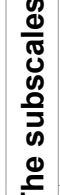 & 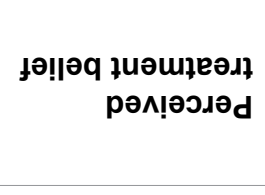 & & 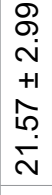 & $\begin{array}{l}\hat{\sigma} \\
i \\
+1 \\
o \infty \\
\infty \\
\infty \\
\dot{N}\end{array}$ & 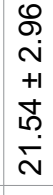 & $\begin{array}{l}\hat{\infty} \\
i \\
+1 \\
+1 \\
i \\
N \\
N\end{array}$ & 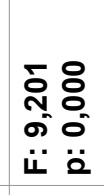 & & 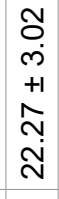 & $\begin{array}{l}8 \\
\stackrel{1}{ } \\
i \\
+1 \\
o \infty \\
0 \\
\dot{N}\end{array}$ & 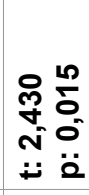 & & $\begin{array}{l}10 \\
\infty \\
i \\
+1 \\
\mathbb{N} \\
\underset{N}{N}\end{array}$ & $\begin{array}{l}0 \\
0 \\
\dot{m} \\
+1 \\
\hat{0} \\
\dot{N} \\
\dot{N}\end{array}$ & $\begin{array}{l}0 \\
1 \\
n \\
+1 \\
+1 \\
8 \\
0 \\
0 \\
\dot{N}\end{array}$ & 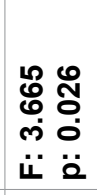 & & $\begin{array}{l}\infty \\
\infty \\
i \\
+1 \\
0 \\
0 \\
\vdots \\
\grave{N}\end{array}$ & $\begin{array}{l}0 \\
0 \\
i \\
+1 \\
\infty \\
0 \\
\dot{N}\end{array}$ & $\begin{array}{l}\mathbb{U} \\
i \\
+1 \\
+1 \\
\tilde{O} \\
\mathbb{N}\end{array}$ & 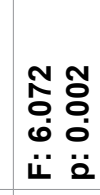 \\
\hline & 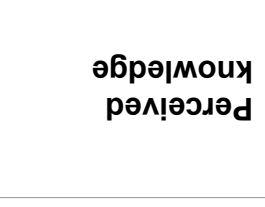 & & 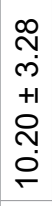 & 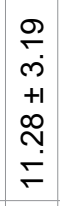 & 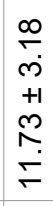 & 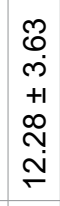 & 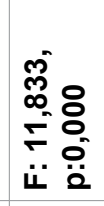 & & $\begin{array}{l}\infty \\
\infty \\
\infty \\
+1 \\
+1 \\
\varnothing \\
\mathscr{T} \\
\leftarrow\end{array}$ & 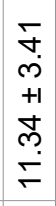 & 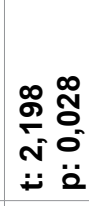 & & 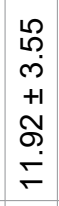 & $\begin{array}{l}0 \\
N \\
m \\
+1 \\
+1 \\
N \\
\stackrel{+}{c} \\
\Gamma\end{array}$ & 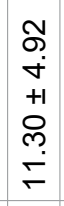 & 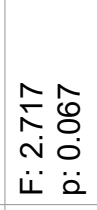 & & $\begin{array}{l}0 \\
0 \\
m \\
+1 \\
+1 \\
\stackrel{N}{N} \\
\vdots \\
0\end{array}$ & 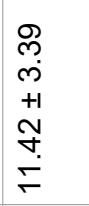 & 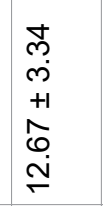 & 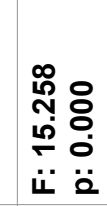 \\
\hline & 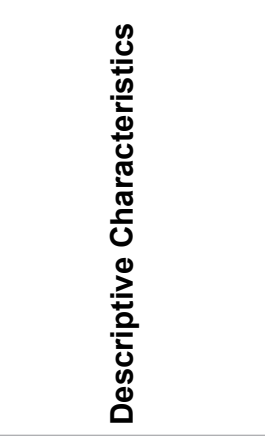 & 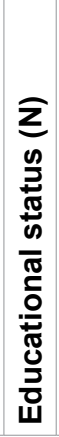 & 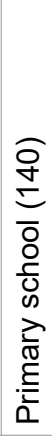 & 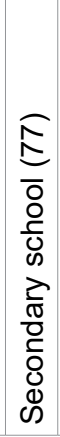 & 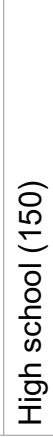 & 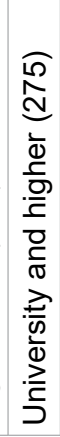 & & 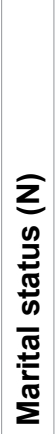 & 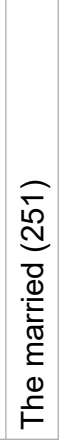 & $\begin{array}{l}\widehat{\bar{\rho}} \\
\frac{0}{0} \\
\frac{0}{0}\end{array}$ & & 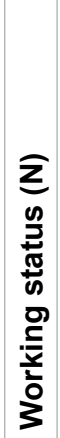 & 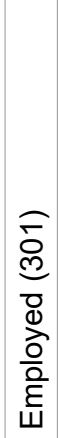 & 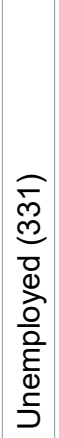 & 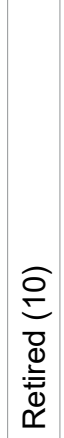 & & 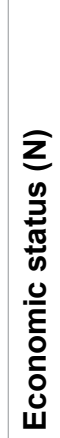 & 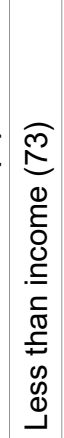 & 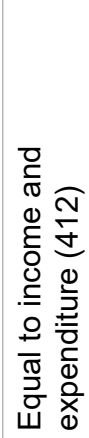 & 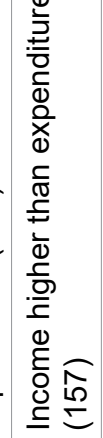 & \\
\hline
\end{tabular}




\begin{tabular}{|c|c|c|c|c|c|c|c|c|}
\hline & $\begin{array}{l}8 \\
0 \\
\dot{0} \\
+1 \\
+1 \\
0 \\
\dot{0} \\
\dot{0}\end{array}$ & $\begin{array}{l}\hat{N} \\
N \\
+1 \\
\infty \\
0 \\
\infty \\
0 \\
0\end{array}$ & 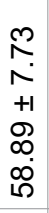 & $\begin{array}{l}\text { స్రి } \\
\infty \\
\infty \\
\ddot{0} \\
\ddot{0}\end{array}$ & & 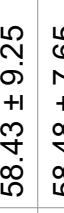 & 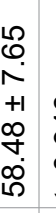 & 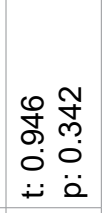 \\
\hline & $\begin{array}{l}\dot{N} \\
+1 \\
\infty \\
\infty \\
\sigma \\
\sigma\end{array}$ & 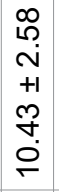 & $\begin{array}{l}\stackrel{L}{L} \\
\stackrel{0}{ } \\
+1 \\
0 \\
0 \\
0 \\
0 \\
0\end{array}$ & 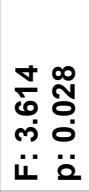 & & 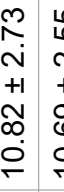 & $\begin{array}{l}+ \\
+1 \\
8 \\
0 \\
0 \\
0\end{array}$ & 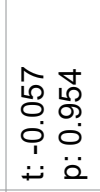 \\
\hline & 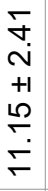 & 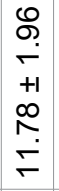 & 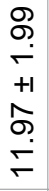 & 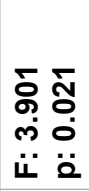 & & 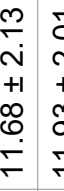 & 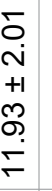 & $\begin{array}{ll} & \\
0 & 0 \\
0 & 0 \\
0 & 0 \\
0 & 0 \\
0 & 0 \\
i-0 & 0\end{array}$ \\
\hline & $\begin{array}{l}0 \\
0 \\
\dot{m} \\
+1 \\
+1 \\
\dot{p} \\
\dot{m}\end{array}$ & 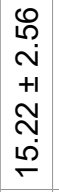 & 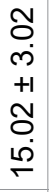 & 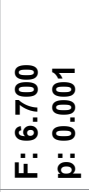 & & 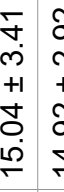 & $\begin{array}{l}N \\
+1 \\
N \\
\tilde{J} \\
\dot{T}\end{array}$ & 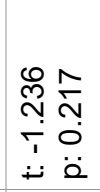 \\
\hline & 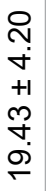 & $\begin{array}{c}\infty \\
\infty \\
\stackrel{1}{ } \\
+1 \\
\stackrel{1}{N} \\
\stackrel{N}{N}\end{array}$ & $\begin{array}{l}\tilde{T} \\
\dot{N} \\
+1 \\
\tilde{O} \\
\dot{\Sigma} \\
\dot{N}\end{array}$ & 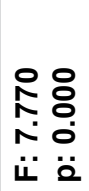 & & 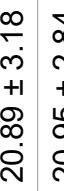 & 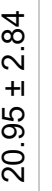 & 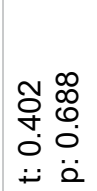 \\
\hline & 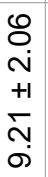 & 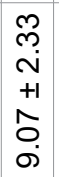 & 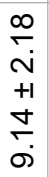 & $\begin{array}{ll} & 0 \\
0 & 0 \\
0 & 0 \\
0 & 0 \\
\ddot{1} & 0\end{array}$ & & 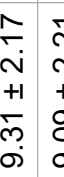 & $\begin{array}{l}\bar{N} \\
\\
+1 \\
\delta \\
\dot{0} \\
\dot{\sigma}\end{array}$ & 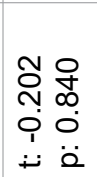 \\
\hline & 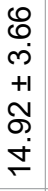 & $\begin{array}{l}0 \\
0 \\
m \\
+1 \\
+1 \\
\tilde{m} \\
\stackrel{b}{n}\end{array}$ & 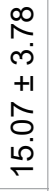 & 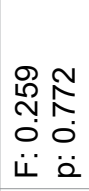 & & 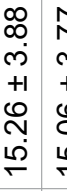 & $\begin{array}{l}\hat{N} \\
\dot{n} \\
+1 \\
\varnothing \\
\dot{\rho} \\
\dot{\rho} \\
\end{array}$ & \begin{tabular}{ll}
8 & 8 \\
$\not 0$ & $\stackrel{2}{0}$ \\
\hdashline & 0 \\
$i$ & 0
\end{tabular} \\
\hline & $\begin{array}{l}\stackrel{0}{c} \\
\dot{m} \\
+1 \\
+1 \\
0 \\
0 \\
0\end{array}$ & $\begin{array}{c}\widehat{c} \\
\infty \\
i \\
+1 \\
+ \\
\tilde{\sigma} \\
\infty\end{array}$ & $\begin{array}{l}\stackrel{8}{ } \\
i \\
+1 \\
+1 \\
\stackrel{9}{+} \\
\infty\end{array}$ & $\begin{array}{l}\infty \\
0 \\
0 \\
\\
\ddot{1} \\
\ddot{1}\end{array}$ & & 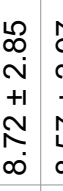 & $\begin{array}{l}\hat{\sigma} \\
i \\
+1 \\
+1 \\
i \\
\infty \\
\infty\end{array}$ & 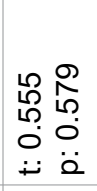 \\
\hline & 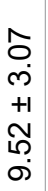 & $\begin{array}{l}0 \\
\tilde{m} \\
\infty \\
+1 \\
\infty \\
\check{c} \\
\stackrel{0}{-}\end{array}$ & 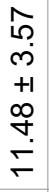 & 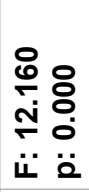 & & 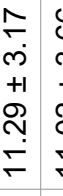 & 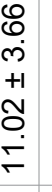 & 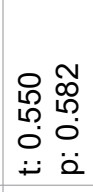 \\
\hline & $\begin{array}{l}0 \\
0 \\
0 \\
0 \\
+1 \\
0 \\
0 \\
0 \\
\dot{N}\end{array}$ & 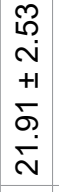 & $\begin{array}{l}8 \\
\dot{j} \\
+1 \\
+1 \\
\text { पे } \\
\text { N }\end{array}$ & 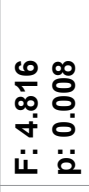 & & 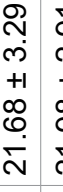 & 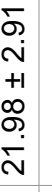 & 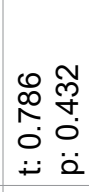 \\
\hline & $\begin{array}{l}0 \\
\infty \\
\dot{m} \\
+1 \\
+1 \\
\infty \\
\infty \\
0 \\
0\end{array}$ & $\begin{array}{l}0 \\
10 \\
0 \\
+1 \\
+1 \\
8 \\
\dot{-} \\
\end{array}$ & 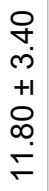 & 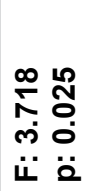 & & $\begin{array}{ll}\bar{m} & \\
\dot{m} & \\
+1 & \\
\infty & \\
\infty & \\
\dot{E} & \\
& \end{array}$ & 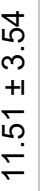 & 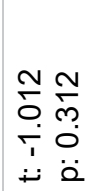 \\
\hline 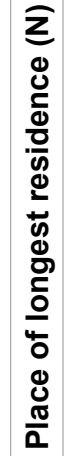 & & 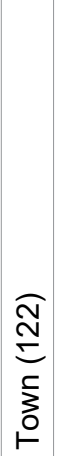 & & & 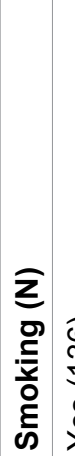 & 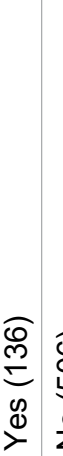 & & \\
\hline
\end{tabular}


Table 5: Regression analysis of the participants' Healthy Life Awareness Scale subscales and Breast Cancer Perception Scale subscales.

\begin{tabular}{|c|c|c|c|c|c|c|c|c|}
\hline \multicolumn{2}{|c|}{ Dependent variables } & \multirow{2}{*}{$\begin{array}{l}\text { Independent variables } \\
\text { Constant }\end{array}$} & \multirow{2}{*}{$\frac{\text { B }}{11.241}$} & \multirow{2}{*}{\begin{tabular}{|c|}
$\mathbf{t}$ \\
10.417
\end{tabular}} & \multirow{2}{*}{$\begin{array}{r}\mathbf{p} \\
0.000\end{array}$} & \multirow[t]{2}{*}{$\mathbf{F}$} & \multirow[t]{2}{*}{ Model (p) } & \multirow[t]{2}{*}{$\mathbf{R}^{2}$} \\
\hline \multirow{7}{*}{ Model 1} & HLAS & & & & & & & \\
\hline & \multirow{4}{*}{ Change } & Perceived knowledge & 0.162 & 4.181 & 0.000 & \multirow{6}{*}{39.345} & \multirow{6}{*}{0.000} & \multirow{6}{*}{0.271} \\
\hline & & Perceived treatment belief & 0.451 & 11.630 & 0.000 & & & \\
\hline & & Perceived need for healthcheck & -0.084 & -2.055 & 0.040 & & & \\
\hline & & Perceived stigma & -0.063 & -1.563 & 0.119 & & & \\
\hline & \multirow{2}{*}{ (D-W:1.753) } & Perceived fear & 0.084 & 2.167 & 0.031 & & & \\
\hline & & Perceived risk & -0.097 & -2.686 & 0.007 & & & \\
\hline \multirow{7}{*}{ Model 2} & HLAS & Constant & 5.309 & 4.364 & 0.000 & \multirow{7}{*}{17.704} & \multirow{7}{*}{0.000} & \multirow{7}{*}{0.143} \\
\hline & \multirow{6}{*}{ Socialization } & Perceived knowledge & 0.212 & 5.048 & 0.000 & & & \\
\hline & & Perceived treatment belief & 0.193 & 4.588 & 0.000 & & & \\
\hline & & Perceived need for healthcheck & 0.123 & 2.782 & 0.006 & & & \\
\hline & & Perceived stigma & 0.047 & 1.090 & 0.276 & & & \\
\hline & & Perceived fear & 0.135 & 3.224 & 0.001 & & & \\
\hline & & Perceived risk & 0.001 & 0.015 & 0.988 & & & \\
\hline \multirow{7}{*}{ Model 3} & HLAS & Constant & 5.158 & 6.762 & 0.000 & \multirow{7}{*}{36.004} & \multirow{7}{*}{0.000} & \multirow{7}{*}{0.254} \\
\hline & \multirow{6}{*}{ Responsibility } & Perceived knowledge & 0.145 & 6.349 & 0.000 & & & \\
\hline & & Perceived treatment belief & 0.265 & 9.938 & 0.000 & & & \\
\hline & & Perceived need for healthcheck & -0.031 & -1.329 & 0.184 & & & \\
\hline & & Perceived stigma & -0.042 & -1.483 & 0.139 & & & \\
\hline & & Perceived fear & 0.012 & 0.578 & 0.563 & & & \\
\hline & & Perceived risk & -0.028 & -0.833 & 0.405 & & & \\
\hline \multirow{7}{*}{ Model 4} & HLAS & Constant & 5.825 & 5.644 & 0.000 & \multirow{7}{*}{19.369} & \multirow{7}{*}{0.000} & \multirow{7}{*}{0.155} \\
\hline & \multirow{6}{*}{$(D-W: 1.688)$} & Perceived knowledge & 0.231 & 5.535 & 0.000 & & & \\
\hline & & Perceived treatment belief & 0.153 & 3.657 & 0.000 & & & \\
\hline & & Perceived need for healthcheck & 0.122 & 2.787 & 0.005 & & & \\
\hline & & Perceived stigma & -0.037 & -0.857 & 0.392 & & & \\
\hline & & Perceived fear & -0.044 & -1.049 & 0.294 & & & \\
\hline & & Perceived risk & -0.023 & -0.588 & 0.557 & & & \\
\hline
\end{tabular}

Notes: D-W: Durbin-Watson test; HLAS: Healthy Life Awareness Scale

knowledge ( $\beta=0.145, p<0.001$ ) and perceived need for treatment $(B=0.265, p<0.001$ ) scores increased, socialization scores increased significantly with an explanatory value of $25.4 \%$ in model no 3 .

In the regression analysis examining the relationship between HLAS responsibility scores and BCPS subdimension scores (Table 5), model no 5 showed a significant difference ( $\left.F: 19.369, R^{2}: 0.155 p<0.001\right)$.

\section{Discussion}

In this study, it was determined that women with higher education levels, an income-generating job and/ or higher economic status perceived breast cancer more positively and had higher levels of awareness of healthy lifestyle. In addition, it was found out that there was a moderately significant positive relationship between the perceived knowledge and perceived treatment belief sub-dimensions in BCPS in women with high awareness of healthy lifestyle.
Education is one of the important determinants of the awareness of healthy lifestyle. In a study reporting results similar to those of this study, it was reported that the awareness of breast cancer increased as the education levels increased. This is because women with higher levels of education are more socialized and may share experiences to a higher extent with other women including friends, relatives, and colleagues [15]. In another study, it was reported that the predictors of undergoing breast cancer screening were affected by several factors including age, occupation, spouse's occupation, and household income [16]. Occupation is one of the determinants of undergoing breast cancer screening. One study reported that a woman working as a servant was $32 \%$ less likely to perform breast self-examinations compared to a whitecollar woman. White-collar women tend to have higher levels of social relationships and higher education levels, and thus, are more knowledgeable about breast screening programs and attend them at higher rates compared to servants [17]. 
The results of this study showed that income level is associated with the perception of breast cancer and the awareness of healthy lifestyle. Similar to the findings of this study, Amornsiripanitch, et al. reported that women with high socioeconomic status were more interested in breast cancer risk assessment and improvement activities [18]. In another study, high rates of breast cancer screening were reported in women having high household incomes [16]. In contrast, a study by Lee, et al. from Korea reported that household income did not affect the breast cancer screening rate. It has been suggested that the impact of household income on participation rates in screening programs might appear low because the Korean government offers free screening services to people with low household income levels [19]. Similarly, breast cancer screening programs are provided free of charge in Turkey. However, it has been determined in this study that perceived knowledge, treatment belief, and awareness of a healthy lifestyle are all affected by some factors such as education, income level, and employment.

Screening is critical for the early diagnosis and treatment of breast cancer, which is one of the most common types of cancer among women all over the world. Studies from various cultures report that several reasons prevent women from undergoing breast cancer screening, including socioeconomic and cultural factors, religious and personal beliefs, lack of knowledge, fear of getting sick as a result of screening, embarrassment about seeing a male doctor, and fear of violation of privacy. In a study conducted in Al Hassa, Saudi Arabia, it was reported that the most commonly perceived barriers to breast cancer screening among women were personal fears, fear of physicians, fear of consequences, and fear of hospitals and health facilities [20]. Such fears must be addressed at the time of starting and implementing breast cancer screening programs through community-based awareness plans, intensive educational campaigns for women based on socio-cultural contexts, and the use of culture-sensitive educational materials, which target the outcomes of such activities and highlight the importance of early detection.

The frequency of breast cancer screening in developing or underdeveloped countries is lower compared to that in developed countries. Therefore, there is a need for more efforts in such countries to improve women's knowledge about breast screening methods and the importance of screening for early detection of breast cancer. It has been reported that $41.1 \%$ of Iranian women undergo clinical breast examinations and $21.7 \%$ undergo mammography, while $82.8 \%$ of German women undergo clinical breast examinations and $55.5 \%$ have a history of undergoing mammography $[16,21]$. In the present study, we found that perceived knowledge and awareness of healthy living increased in parallel to education and income levels. In this context, it can be suggested that especially in developing and underdeveloped countries, women with low socioeconomic, educational, and income status should receive further attention so that their perception of breast cancer and awareness of a healthy lifestyle can be improved.

A study conducted in Nigeria investigated the perceived risk of breast cancer and its effects on screening behavior in 992 women residing in rural, urban, and semi-urban communities from five randomly selected local government regions. The study reported that women's screening behaviors were affected by perceived risks and determined by their religious and socio-cultural beliefs. The study also reported that the knowledge and awareness of breast cancer in Nigerian women were not sufficient to perceive breast cancer as a threat and women's attitudes towards breast cancer were mostly influenced by the family history of and personal beliefs about breast cancer [22]. In this context, the socio-cultural environment and religious characteristics of women can be taken into account when developing interventions to promote breast cancer screening. In another study, a relationship was reported between rural residence among other factors and delays in patients' presenting to healthcare services. In the study, the limited access to health services by rural residents was reported as the reason for delays in treatment [23]. In the present study, it was also determined that women living in urban areas had higher perceived knowledge, perceived treatment belief, and perceived need for a health check, and lower perceived stigma sub-dimension scores in BCPS and higher HLAS scores compared to the scores from those living in rural areas. There is a need for programs to be implemented by the government aiming to offer equal healthcare services to all women regardless of whether they live in urban or rural areas. Although breast cancer screening services are offered to women free of charge in Turkey, it can be suggested that the development of further programs to improve access to urban hospitals and healthcare services is still needed for women living in rural areas.

In the present study, it was determined that unmarried women had higher levels of perceived stigma and fear of breast cancer compared to married women. Amin, et al. reported that unmarried women had lower breast cancer awareness levels compared to other participants and it might be because such women were younger or less interested in sharing personal experiences with other women [15]. In another study, it was reported that there might be important reasons for women to hide or delay help-seeking behavior for breast masses because of the involvement of several factors including social rejection, stigma, and fear of losing the spouse [23]. It can be suggested that this is because women might have had concerns of not being able to find a spouse, losing the spouse, not being able to 
have children, or not being able to breastfeed. Indeed, women might associate the breast with the perception of femininity or sexuality and the abovementioned fears might be aggravated by inadequate awareness, knowledge, education, and socioeconomic levels. In this context, we believe that the significance of the breast to women should be understood well to evaluate women's perceptions of breast cancer and relevant programs addressing the values and beliefs of the society should be developed.

Several studies reported various results about the factors predicting breast cancer screening behaviors. Hasani, et al. reported that perceived benefits in the health belief model and self-efficacy were the predictors of breast self-examination in women admitted to health centres in Bandar Abbas [24]. The study performed by Lee, et al. found out that the anxiety of developing breast cancer and the persisting high levels of anxiety resulted in a lower quality of life during the waiting period before receiving the screening results [25].

Another study showed that women's self-efficacy, perceived benefits levels, and perceived barriers levels could predict breast self-examination behavior. Therefore, educational interventions should be considered to improve health behaviors and skills to promote breast self-examinations. It is also necessary to better understand the benefits of healthy lifestyle behaviors and remove barriers to such behaviors through proper education. Furthermore, appropriate planning is recommended for the implementation of educational interventions to promote screening programs [26]. Another study reported that educational interventions have the potential to promote breast cancer awareness. Such educational activities address symptoms and age- and lifestyle-related risks of breast cancer and aim to improve breast examination behaviors [27]. Nurses can provide ongoing psychosocial support to improve breast cancer awareness and assist women to seek professional advice when needed. In order to reduce the total disease burden of breast cancer, healthcare providers should address factors, which affect women's behaviors toward screening tests, as an efficient education method. Since this study was carried out only in Istanbul, the generalizability of the study results is limited.

\section{Conclusion}

It has been demonstrated in this study that women with advantages of high educational and economic levels, an income-generating job, and working in urban areas have higher perceived knowledge and treatment belief levels and low levels of fear with regard to breast cancer. Nurses providing care for breast diseases and screening should take into consideration that women with low socioeconomic levels need to be provided with educational and awareness activities. Furthermore, it was found out in this study that unmarried women had higher scores of perceived stigma, fear, and risk compared to married women. This finding should be remembered by nurses providing care to such patients. We may further suggest that programs should be developed to improve the quality of psychosocial and holistic care provided by nurses and women's perceptions and awareness of breast cancer.

\section{References}

1. Walker SN, Sechrist KR, Pender NJ (1987) The HealthPromoting Lifestyle Profile: development and psychometric characteristics. Nurs Res 36: 76-81.

2. Brown KW, Ryan RM (2003) The benefits of being present: mindfulness and its role in psychological well-being. J Pers Soc Psychol 84: 822-848.

3. Özer E, Yılmaz N (2020) Healthy Life Awareness: A Scale Development Study. J Tradit Complem Med 3: 47-60.

4. Owen FK, Çelik ND (2018) Lifelong Healthy Lifestyle and Wellness. Current Approaches in Psychiatry 10: 440-453.

5. Song M, Giovannucci E (2016) Preventable incidence and mortality of carcinoma associated with lifestyle factors among white adults in the United States. JAMA Oncology 2: 1154-1161.

6. Anand P, Kunnumakara AB, Sundaram C, Harikumar KB, Tharakan ST, et al. (2008) Cancer is a preventable disease that requires major lifestyle changes. Pharm Res 25: 20972116.

7. Arnuna P, Zotor FB (2008) Epidemiological and nutrition transition in developing countries: Impact on human health and development. Proc Nutr Soc 67: 82-90.

8. Danaei G, Ding EL, Mozaffarian D, Taylor B, Rehm J, et al. (2009) The preventable causes of death in the United States: comparative risk assessment of dietary, lifestyle, and metabolic risk factors. PLoS Med 6: e1000058.

9. Bray F, Ferlay J, Soerjomataram I, Siegel RL, Torre LA, et al. (2018) Global cancer statistics 2018: GLOBOCAN estimates of incidence and mortality worldwide for 36 cancers in 185 countries. CA Cancer J Clin 68: 394-424.

10. Özmen V, Özmen T, Doğru V (2019) Breast cancer in Turkey: Analysis of 20.000 patients with breast cancer. Eur J Breast Health 15: 141-146.

11. TR Ministry of Health (2020) Health Services General Directorate, Breast Cancer Prevention, Screening, Diagnosis, Treatment and Follow-up Clinical Guidelines ISBN: 978-975-590-771-0.

12. Colditz GA, Bohlke K (2014) Priorities for the primary prevention of breast cancer. CA Cancer J Clin 64: 186-194.

13. Taylan S, Özkan I, Adıbelli D (2021) Breast Cancer Perception Scale: Psychometric Development Study. Eur J Breast Health 17: 95-102.

14. Tunç A, Atılgan A (2017) An administrative understanding on perception: Perception administration. International Journal of Disciplines Economics \& Administrative Sciences Studies 3: 228-238.

15. Amin MN, Uddin MG, Uddin MN, Rahaman MZ, Siddiqui SA, et al. (2020) A hospital based survey to evaluate knowledge, awareness and perceived barriers regarding breast cancer screening among females in Bangladesh. Heliyon 6: e03753. 
16. Kardan-Souraki M, Moosazadeh M, Khani S, Hamzehgardeshi Z (2019) Factors related to breast cancer screening in women in the northern part of Iran: A crosssectional study. Open Access Maced J Med Sci 7: 637-642.

17. Soltanahmadi, Z, Abbaszadeh, A, Tirgari B (2010) A survey on the rate and causes of women's participation or nonparticipation in breast and cervical cancers screening programs. The Iranian Journal of Obstetrics, Gynecology and Infertility 13: 37-46.

18. Amornsiripanitch N, Ameri SM, Goldberg RJ (2021) Impact of age, race, and socioeconomic status on women's perceptions and preferences regarding communication of estimated breast cancer risk. Academic Radiology 28: 655663.

19. Lee K, Lim HT, Park SM (2010) Factors associated with use of breastcancer screening services by women aged $\geq$ 40 years in Korea:The Third Korea National Health and Nutrition Examination Survey 2005 (KNHANES III). BMC Cancer 10: 144.

20. Abdel-Aziz SB, Amin TT, Al-Gadeeb MB, Alhassar Al, Al-Ramadan A, et al. (2017) Perceived barriers to breast cancer screening among Saudi women at primary care setting, Asian Pacific Journal of Cancer Prevention 18: 2409-2417.

21. Klug SJ, Hetzer M, Blettner M (2005) Screening for breast and cervical cancer in a large German city: participation, motivation and knowledge of risk factors. Eur J Public Health 15: 70-77.
22. Allo T, Edewor P, Imhonopi D (2018) Knowledge, attitude and behavioural disposition of Nigerian women towards breast cancer. Proceedings of ADVED 2018- 4th International Conference on Advances in Education and Social Sciences, 15-17 October-Istanbul, Turkey.

23. Sayed S, Ngugi AK, Mahoney MR, Kurji J, Talib ZM, et al. (2019) Breast Cancer knowledge, perceptions and practices in a rural Community in Coastal Kenya. BMC Public Health 19: 1-13.

24. Hasani L, Aghamolaei T, Tavafian SS, Zare SH (2011) Constructs of the health belief model as predicting factors in breast self-examination. HAYAT-Journal of Faculty of Nursing and Midwifery 17: 62-69.

25. Lee JM, Lowry KP, Chubiz JEC, Swan JS, Motazedi T, et al. (2020) Breast cancer risk, worry, and anxiety: Effect on patient perceptions of false-positive screening results. The Breast 50: 104-112.

26. Darvishpour A, Vajari SM, Noroozi S (2018) Can health belief model predict breast cancer screening behaviors? Open Access Maced J Med Sci 6: 949-953.

27. Kennedy C (2017) Interventions for raising breast cancer awareness in women. International Journal of Nursing Practice 24: e12582. 